\title{
miR-221-3p Delivered by BMMSC-Derived Microvesicles Promotes the Development of Acute Myelocytic Leukemia
}

\author{
Xuewu Zhang ${ }^{\dagger}, Y u X^{\dagger}{ }^{\dagger}$, Jinghan Wang, Shuqi Zhao, Jianhu Li, Xin Huang, Huan Xu, \\ Xiang Zhang, Shanshan Suo, Yunfei Lv, Yi Zhang and Wenjuan Yu* \\ Department of Hematology, Zhejiang University School of Medicine First Affiliated Hospital, Hangzhou, China
}

OPEN ACCESS

Edited by:

Tao Huang,

Shanghai Institutes for Biological

Sciences (CAS), China

Reviewed by:

Jinhang Zhu,

Shanghai Jiao Tong University, China

Minzhi Zhao,

National Center for Nanoscience and

Technology (CAS), China

${ }^{*}$ Correspondence:

Wenjuan Yu

1306023@zju.edu.cn

tThese authors have contributed equally to this work

Specialty section:

This article was submitted to Bioinformatics and Computational

Biology,

a section of the journal Frontiers in Bioengineering and

Biotechnology

Received: 20 December 2019 Accepted: 29 January 2020

Published: 14 February 2020

Citation:

Zhang $X, X U Y$, Wang J, Zhao S, Li J, Huang $X, X u H$, Zhang $X$, Suo S, LV Y, Zhang $Y$ and Yu W (2020) miR-221-3p Delivered by BMMSC-Derived

Microvesicles Promotes the Development of Acute Myelocytic Leukemia. Front. Bioeng. Biotechnol. 8:81. doi: 10.3389/fbioe.2020.00081
Objective: The study aims to investigate the effects of miR-221-3p in bone marrow mesenchymal stem cell (BMMSC)-derived microvesicles (MVs) on cell cycle, proliferation and invasion of acute myelocytic leukemia (AML).

Methods: Bioinformatics was used to predict differentially expressed miRNAs (DEmiRNAs) in AML. The morphology of BMMSC-derived MVs was observed under an electron microscope, and the positional relation of MVs and OCl-AML2 cells was observed by a fluorescence microscope. MTT, Transwell, and flow cytometry assays were used to analyze the effects of MVs on OCl-AML2 cells. The targeted relationship between miR-221-3p and CDKN1C was detected by dual luciferase assay.

Results: It was verified that miR-221-3p promoted the proliferation, invasion and migration of OCl-AML2 cells, and induced the cell cycle arrest in G1/S phase as well as inhibited cell apoptosis. Further studies showed that MVs promoted the proliferation, migration and invasion of AML, and induced the cell cycle arrest in G1/S phase through miR-221-3p. It was confirmed that miR-221-3p can directly target CDKN1C to regulate cell cycle, proliferation and invasion of AML.

Conclusion: miR-221-3p in BMMSC-derived MVs regulated AML cell cycle, cell proliferation and invasion through targeting CDKN1C. miR-221-3p and CDKN1C were considered to be potential targets and biomarkers for the treatment of AML in clinic.

Keywords: BMMSC, microvesicles, miR-221-3p, AML, cell proliferation and invasion, cell cycle

\section{INTRODUCTION}

Acute myelocytic leukemia (AML) is a malignant tumor of abnormal clonal in immature myeloid hematopoietic cells with high heterogeneity, which is characterized by differentiation and maturation disorders along with block of apoptosis in clonal hematopoietic stem cells or progenitor cells, leading to malignant proliferation and accumulation of cells in the bone marrow, thus affecting normal hematopoiesis (Coombs et al., 2016; Khwaja et al., 2016). AML is the most-common acute leukemia in adults, but it predominantly occurs in older people ( $>60$ years of age), with a median age at diagnosis of 67 (Coombs et al., 2016). It typically presents with a rapid onset of symptoms that are attributable to bone marrow failure and may be fatal within weeks or months when left untreated. Currently, chemotherapy and hematopoietic stem cell transplantation 
are the main treatments, but the success rate of AML cure remains low (Cornelissen and Blaise, 2016; Stein and Tallman, 2016). Therefore, it is particularly important to study the pathogenesis of AML and explore the possible therapeutic approaches.

Microvesicles (MVs) are extracellular vesicles between $100 \mathrm{~nm}$ and $1 \mu \mathrm{m}$ that derived from normal cells and cancer cells. MVs can transfer proteins, glycoproteins, lipids, nucleic acids, and cytokines from maternal cells to recipient cells, promoting phenotype changes of recipient cells and playing an important role in intercellular communication (Hansen et al., 2014; Gopal et al., 2017; Abbasian et al., 2018). Studies have found that tumor-derived MVs (TMV) can interact directly with tumor cells and play a macro-messenger role to promote the transfer of molecular substances between tumor cells to facilitate tumor growth (Stec et al., 2015a,b). MVs derived from bone marrow mesenchymal stem cell (BMMSC) can promote tumorigenesis and development (Crompot et al., 2017; Boyiadzis and Whiteside, 2018). miRNAs in MVs, as post-transcriptional regulatory elements, directly regulate gene expression, target mRNA expression and translation or induce mRNA degradation to reduce protein synthesis by directly binding with the $3^{\prime}$-untranslation region ( $3^{\prime}$-UTR) of specific mRNA targets (Braicu et al., 2015; Jerez et al., 2019), ultimately induce multiple pathophysiological processes, such as leukemia stem cell formation, regulation of tumor cell proliferation, angiogenesis, invasion, metastasis, and immune escape to modulate leukemia development (Braicu et al., 2015; Del Principe et al., 2017).

microRNAs (miRNA) are a class of evolutionarily conserved 22 to 24-nucleotide small RNAs in length, which are widely found in eukaryotic cells with molecular functions to regulate cell differentiation, proliferation and apoptosis (Summerer et al., 2013; Zheng et al., 2013). miR-221-3p has important regulatory effects on a variety of cancers as an important miRNA. Studies have reported that in cervical squamous cell carcinoma, miR221-3p in MVs promotes lymph angiogenesis and lymphatic metastasis by targeting VASH1 (Zhou et al., 2019), and promotes angiogenesis by targeting THBS2 (Wu et al., 2019). We previously found that miR-221-3p was significantly highly expressed in AML patients through bioinformatics, and miR221-3p mainly existed in BMMSC-derived MVs. These results suggest that miR-221-3p in BMMSC-derived MVs has certain regulatory effects on AML cells.

Therefore, in this paper, we explored the regulatory effects and mechanism of miR-221-3p in BMMSC-derived MVs on cell cycle, cell proliferation and invasion of AML through in vitro experiments, so as to further understand the pathogenesis of AML and provide new ideas for future clinical diagnosis and treatment.

\section{MATERIALS AND METHODS}

\section{Cell Lines and Patients}

Normal human BMMSCs were purchased from Kunming cell bank, Chinese Academy of Sciences (No. 3153C0001000000244). BMMSCs were isolated from AML patients and human AML cells OCI-AML2 (BNCC341618) were purchased from BeNa Culture Collection (China).

Fifteen AML patients and 18 control samples (peripheral blood or bone marrow) were obtained with the informed consent of the patient or healthy subject and were collected at the First Affiliated Hospital of Zhejiang University through the protocol approved by the review committee.

\section{Bioinformatics Analysis}

AML-related miRNA expression dataset GSE49665 was obtained from GEO database (https://www.ncbi.nlm.nih.gov/geoprofiles/) to screen differentially expressed miRNAs (DEmiRNAs) and determine target miRNAs. Target miRNAs were found to be highly expressed in the MVs of fiber cells and mesenchymal stem cells (MSCs) via searching expression location In the EV miRNA database (http://bioinfo.life.hust.edu.cn/EVmiRNA). The downstream target genes of the target miRNAs were predicted by TargetScan database (http://www.targetscan.org/vert_72/), miRSearch database (https://www.exiqon.com/miRSearch), and mirDIP database (http://ophid.utoronto.ca/mirDIP/index.jsp), and differential analysis was conducted on AML gene expression in TCGA. The down-regulated genes in AML were selected to intersect with the predicted downstream target genes. Finally, the target genes with the most significant expression changes were detected by signaling pathway enrichment analysis.

\section{Isolation, Culture and Analysis of BMMSC}

BMMSCs were obtained by density gradient centrifugation. The bone marrow fluids were centrifuged at $1,000 \mathrm{rpm}$ for $10 \mathrm{~min}$, while the lipids and supernatant were absorbed and discarded. The remaining marrow fluids were added with equal quantity of PBS buffer and mixtured, centrifuged at 1,000 rpm for $10 \mathrm{~min}$, and the supernatant was discarded. Then cell suspensions were prepared with $2 \mathrm{~mL}$ PBS buffer at a density of $4 \times 10^{7}$ cells, carefully superimposed on $5 \mathrm{~mL}$ Percoll separation solution (at a density of $1.077 \mathrm{~g} / \mathrm{mL}$ ), and centrifuged at 2,300 rpm for $30 \mathrm{~min}$. After centrifugation, the liquids from top to bottom are: platelet and plasma diluent layer, yellow-brown annular cloudlike mononuclear cell layer, lymphocyte separation liquid layer, red blood cells and granulocyte layer. The mononuclear cell layer was absorbed and mixed with PBS buffer at a ratio of 1:2, and then centrifuged at $1,500 \mathrm{rpm}$ for $10 \mathrm{~min}$. All centrifugations were carried out at room temperature. The supernatant was discarded and cells were washed twice. $1 \times 10^{6}$ cells $/ \mathrm{mL}$ were inoculated in a $25 \mathrm{~cm}^{2}$ culture bottle with $5 \mathrm{~mL}$ BMMSCs medium (containing $10 \%$ fetal bovine serum, FBS). After 2-3 days, non-adhesive cells were removed, and monolayer adherent cells were spread to 70 $80 \%$ of the bottom of the culture bottle. Cells were then isolated in a trypsin solution $(0.25 \%$ trypsin/0.1\% EDTA PBS solution, free of magnesium/magnesium and phenolic red) (Aurogene, Rome, Italy) and re-inoculated at a density of $3.5 \times 10^{3} \mathrm{cells} / \mathrm{cm}^{2}$. The 3-5 generation cells were used for the experiment. Cell growth was analyzed by direct cell count at every passage.

\section{Isolation and Identification of MVs}

BMMSC-derived MVs were isolated using the exoEasy Maxi Kit (qiagen, Germany) according to the manufacturer's instructions. 
MVs were observed by Philips CM120 BioTwin transmission electron microscope (FEI, USA).

\section{Inhibition/Overexpression of miRNA and mRNA}

miR-221-3p inhibitor, $100 \mathrm{nmol} / \mathrm{L}$ miR-221-3p mimic, 100 $\mathrm{nmol} / \mathrm{L}$ overexpression of $\mathrm{CDKN} 1 \mathrm{C}$ and the corresponding negative control (NC) were purchased from GenePharma (Shanghai, China). Approximately $1 \times 10^{5}$ cells were inoculated into 12-well plates during transfection. CDKN1C, miR-221$3 \mathrm{p}$ and negative control were transfected into the cells using LipoFiter kit (Hanbio, Shanghai, China) according to the kit instructions. RNA and proteins were extracted $48 \mathrm{~h}$ after transfection. The sequences of synthesized primers were shown in Supplement Table 1.

\section{Construction of Lentivirus Expression Vector and Cell Transfection}

Human miR-221-3p sequences were amplified and then bound to pcDNA3.1 (+) to form miR-221-3p expression vector (GenePharma, ShangHai, China). PcDNA3.1 carrier was used as blank control. Lentivirus coated miR-221-3p or blank lentivirus was transfected into OCI-AML2 cells and cultured for $96 \mathrm{~h}$ and then treated with puromycin for 4 weeks to screen cells (Santa Cruz organisms).

\section{qRT-PCR}

Total RNA was extracted from tissues and cells using Trizol (Invitrogen) according to the manufacturer's protocol. cDNA was synthesized using reverse transcription system kit (Invitrogen). qRT-PCR was performed on ABI 7900HT instrument (Applied Biosystems, USA). Quantitative PCR was performed using the miScript SYBR Green PCR Kit (Qiagen, Germany) under the following thermal cycling conditions: pre-denaturation at $95^{\circ} \mathrm{C}$ for $10 \mathrm{~min}$, followed by 40 cycles of denaturation at $95^{\circ} \mathrm{C}$ for $2 \mathrm{~min}$, annealing at $95^{\circ} \mathrm{C}$ for $5 \mathrm{~s}$ and extending at $60^{\circ} \mathrm{C}$ for $30 \mathrm{~s}$. CDKN1C was normalized with $\beta$-Actin as an internal reference, and miR-221-3p was normalized with U6 as an internal reference. The relative expression of the target gene mRNAs in the control group and the experimental group were analyzed by $2^{-\Delta \Delta \mathrm{Ct}}$ method. The primers used in the experiment were shown in Supplement Table 1.

\section{Western Blot}

Forty-eight hours after transfection of cells from different treatment groups, the cells were washed three times with cold PBS (Thermo fisher, USA), and lysed on ice using whole protein lysate for $10 \mathrm{~min}$. BCA quantitative kit (Thermo fisher, USA) was used for protein quantification, then $10 \mu \mathrm{l}$ loading buffer was added and proteins were boiled at $95^{\circ} \mathrm{C}$ for $10 \mathrm{~min}$. the proteins were loaded onto SDS-PAGE at $100 \mathrm{~V}$ and transferred to the NC membrane blocked with 5\% BSA/TBST for $60 \mathrm{~min}$. The membrane was incubated with primary antibodies at $4{ }^{\circ} \mathrm{C}$ overnight and then washed with $1 \times$ TBST solution (Solarbio, Beijing, China) at room temperature for $5 \mathrm{~min} \times 3$ times. the membrane was probed with HRP labeled goat-anti-rabbit IgG at room temperature for $120 \mathrm{~min}$, and washed by TBST for three times. After each 20 min, the ECL kit (Solarbio, Beijing, China) was used for detecting luminescence reaction, and the protein blot was photographed and observed. The antibodies used in experiment were listed in Supplement Table 2.

\section{MTT Assay}

OCI-AML 2 cells $\left(5 \times 10^{3}\right.$ cells/100 ul) were seeded into 96 -well plates. Each group was made in triplicate. Proliferation of cells were evaluated by sterile MTT solution (Beyptime) according to the instructions after culture for $12,24,48$, and $72 \mathrm{~h}$, respectively. Absorbance at $490 \mathrm{~nm}$ was measured using a spectrophotometer (Molecular Devices, Sunnyvale, CA, USA).

\section{Transwell Assays}

Transwell migration assay was used to evaluate the migration ability of OCI-AML2 cells. 24-well Transwell Chambers $(8 \mu \mathrm{m}$ aperture, BD Biosciences) were used. For migration assay, cells at a density of $1 \times 10^{5}$ cells/chamber were seeded into the upper chamber and the $600 \mu \mathrm{L}$ of medium containing 10\% FBS (Thermo fisher, USA) was placed in the lower chamber. For invasion assay, $\sim 2 \times 10^{4}$ cells/chamber were seeded in the upper chamber, which was coated with Matrigel. Dulbecco's modified Eagle culture medium (DMEM) containing 10\% FBS (Thermo fisher, USA) was filled into the lower chamber. After incubation at $37^{\circ} \mathrm{C}$ for $48 \mathrm{~h}$, the cells that were not migrated/invaded were cleared away with a cotton swab and the migrated/invaded cells on the lower side were stained with $0.5 \%$ crystal violet. Cells were observed under a microscope, and photographed.

\section{Flow Cytometry (FCM)}

Cell cycle detection: OCI-AML2 cells in growth phase were added with $3 \mathrm{~mL}$ PBS and harvested with $1 \mathrm{~mL}$ trypsin for 1-5 min after removing the liquid. The cell suspension was prepared by adding $5 \mathrm{~mL}$ PBS, and then transferred to a $15 \mathrm{~mL}$ centrifuge tube for centrifugation at 1,500 rpm for $5 \mathrm{~min}$ to discard the supernatant. Five hundred microliters PBS was added for cell suspension, and $2 \mathrm{~mL}$ of cold ethanol of $95 \%$ at $20^{\circ} \mathrm{C}$ was added to the suspension. After mixing, the suspension was fixed for $30 \mathrm{~min}$. Five milliliters PBS was added and centrifuged at 1,500 rpm for $5 \mathrm{~min}$ to remove the supernatant and then added with $5 \mathrm{~mL}$ PBS and centrifuged at $1,500 \mathrm{rpm}$ for $5 \mathrm{~min}$ to discard the supernatant. The cells were stained with $800 \mu \mathrm{L}$ PI at room temperature for $30 \mathrm{~min}$ in darkness. Cell cycle was detected by FCM.

\section{Dual Luciferase Assay}

In order to determine the binding probability of miR-221-3p and $3^{\prime} \mathrm{UTR}$ of CDKN1C, a psiCHECK luciferase reporter vector (Sangon Co., LTD, ShangHai, China) was inserted into 3'UTR of CDKN1C wild type (WT) and mutated type (MUT). HEK293T cells (Thermo fisher, USA) were then inoculated in a 48-well plate and cultured for $24 \mathrm{~h}$. miR-221-3p/NC and psiCHECK WT/MUT plasmids were co-transfected into cells. Finally, luciferase activity was measured by luciferase assay reagent (Promega, Fitchburg, WI, USA).

\section{Statistical Analysis}

All data were processed by SPSS 22.0 statistical software. The measurement data were expressed as mean \pm standard deviation. 


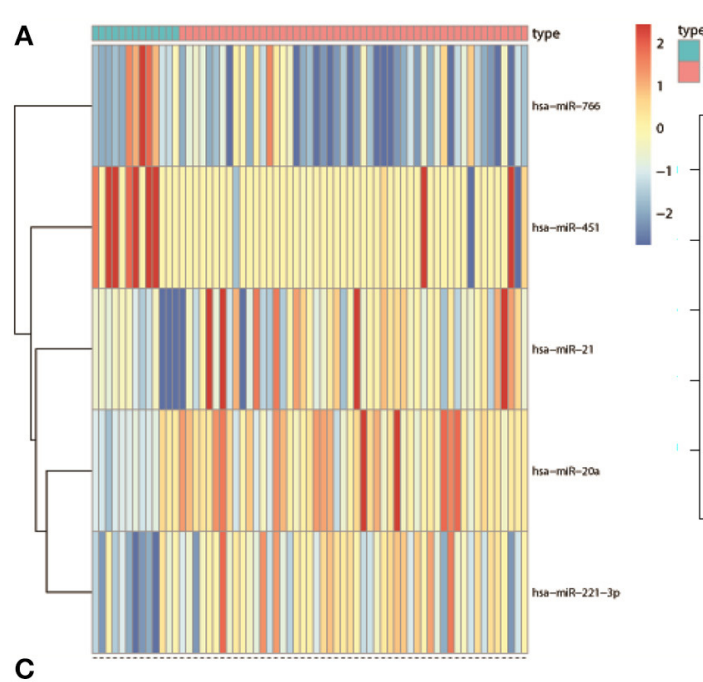

B Tumar

hsa-miR-221-3p Expression of TCGA

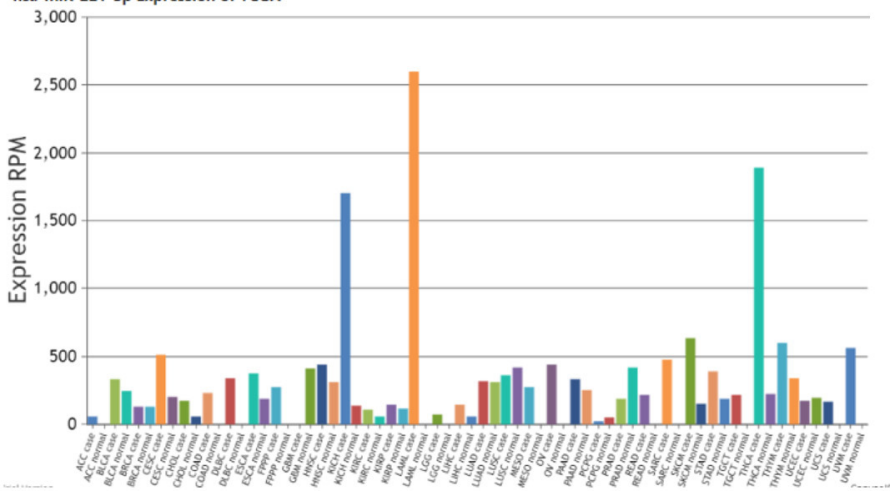

D


FIGURE 1 | miR-221-3p is highly expressed in peripheral blood of patients with leukemia. (A) Heat map of DEmiRNAs in GSE49665 dataset. (B) Boxplot of DEmiRNA. (C) Expression levels of miR-221-3p in different cancer species in TCGA database. (D) miR-221-3p was significantly overexpressed in AML patients.

The comparison between the two groups was analyzed by $t$-test, in which * stood for $P<0.05$.

\section{RESULTS}

\section{miR-221-3p Is Highly Expressed in Peripheral Blood of Patients With AML}

Bioinformatics analysis found that in the miRNA expression dataset GSE49665 of AML patients in the GEO database, 5 DEmiRNAs were obtained and the expression of miR221-3p changed most significantly (Figures 1A,B). At the same time, we detected the expression level of miR-221-3p in various cancers in TCGA database and found that its expression was most significant in AML (Figure 1C), so we chose miR-221-3p for follow-up study. In order to further confirm the high expression of miR-221-3p in the peripheral blood of AML patients, we used qRT-PCR to detect the expression of miR-221-3p in the peripheral blood of 15 normal people and $18 \mathrm{AML}$ patients, and discovered that miR-221-3p was highly expressed in the peripheral blood of AML patients (Figure 1D), which was consistent with the bioinformatics results.

\section{miR-221-3p Regulates AML Cell Cycle, Proliferation, and Invasion}

miR-221-3p was overexpressed in OCI-AML2 cells (Figure 2A) to further explore its role in AML. Analysis of MTT (Figure 2B) and Transwell (Figure 2C) assays revealed that overexpression of miR-221-3p could significantly improve the viability, migration and invasion abilities of OCI-AML2 cells. FCM assay were performed on NC-mimic and oe-miR-221-3p OCI-AML2 cells. The results indicated that overexpression of miR-221-3p reduced the number of cells in the G0/G1 phase with the number of cells in the divisions increased in OCI-AML2 cells (Figure 2D). The expressions of PARP, caspase 8 , cleave caspase 8 , caspase 9 , and other apoptosis-related proteins detected by western blot were decreased after overexpression of miR-221-3p (Figure 2E), indicating that overexpression of miR-221-3p weakened the apoptosis of OCI-AML2 cells, and the results were consistent with FCM.

\section{BMMSC-Derived MVs Regulate the Function of AML Cells}

At present, studies have found that miRNAs can be produced by other cells and transported to target cells through MVs for 

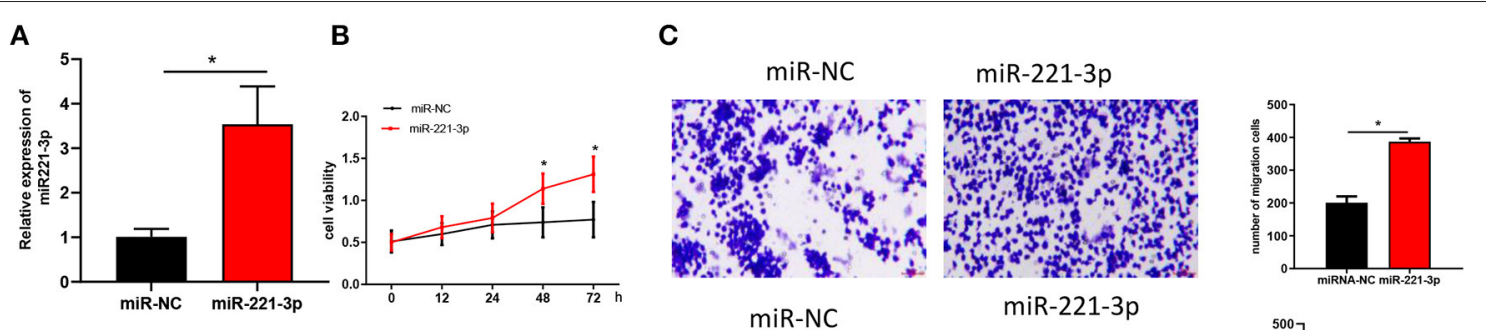

D $\quad$ miR-NC
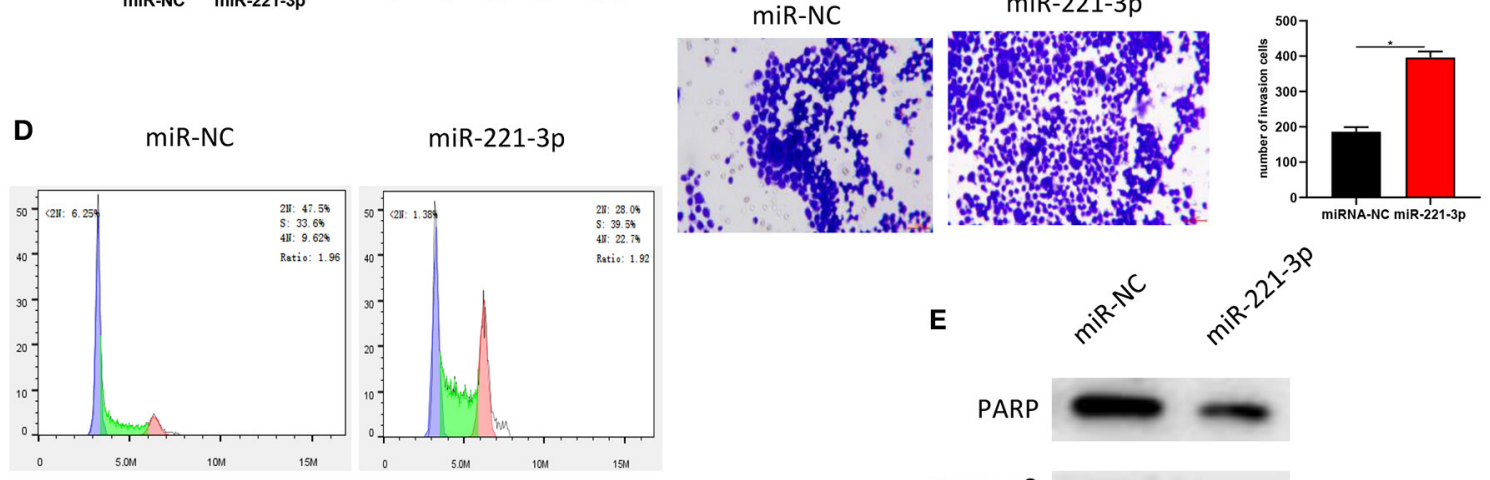

$\operatorname{miR}-221-3 p$
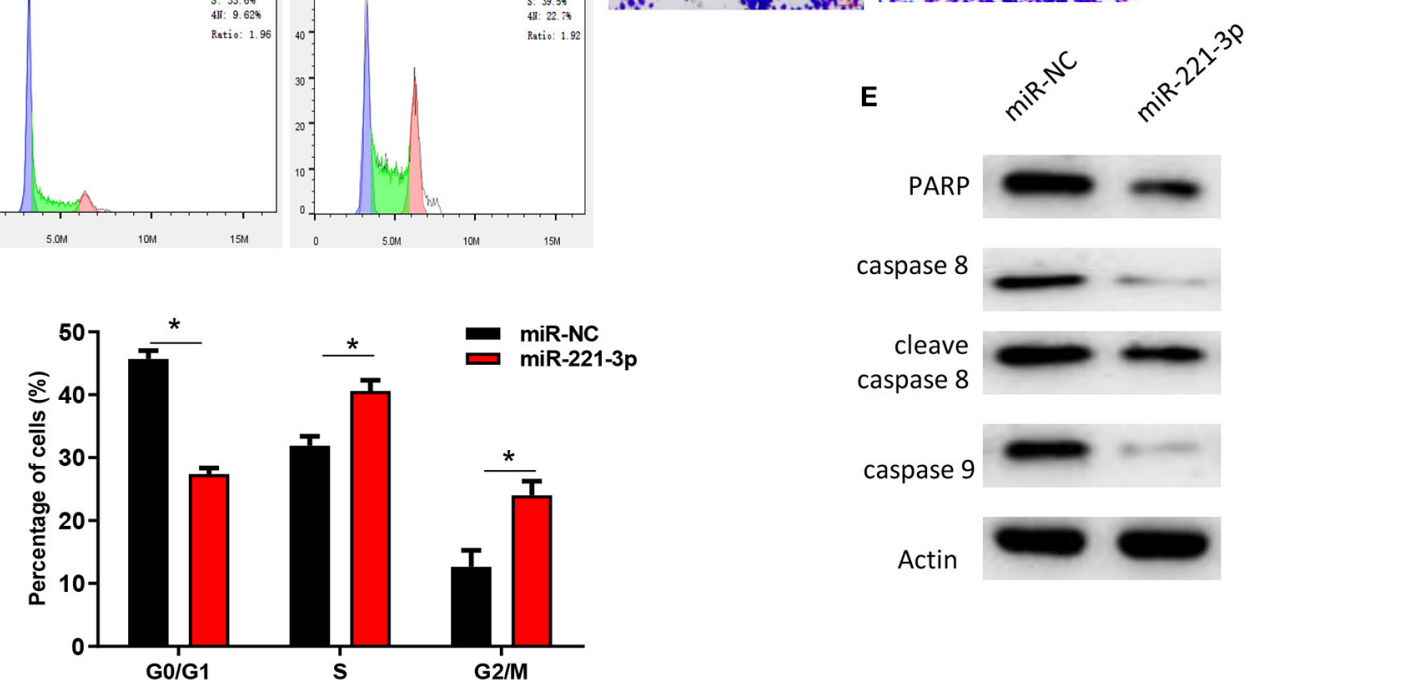

FIGURE 2 | miR-221-3p regulates AML cell cycle, proliferation, and invasion. (A) miR-221-3p expression in each group. (B) The effect of miR-221-3p overexpression on the activity of OCI-AML2 cells was tested by MTT. (C) The effects of miR-221-3p overexpression on invasion and migration of OCl-AML2 cells were detected by Transwell assay $(100 \times)$. (D) The effects of overexpression of miR-221-3p overexpression on the cell cycle of OCI-AML2 was detected by FCM. (E) The effect of miR-221-3p overexpression on expressions of apoptosis-related proteins in OCl-AML2 cells.

further function (Momen-Heravi et al., 2015; Hornick et al., 2016; Lu, 2017), so we speculated that miR-221-3p may be carried by MVs to act on AML cells and thus exerting its regulatory role. We searched the expression location of miR221-3p in the EVmiRNA database and found that its content in MVs of fibroblast and MSCs was significantly higher than that in MVs of other cells (Figure 3A). Meanwhile, studies have reported that BMMSC can affect the morphology, adhesion and microenvironment of leukemia stem cells (Roversi et al., 2019). Then we hypothesized that miR-221-3p was contained in the BMMSC-derived MVs and entered the blood to affect the morphological function of AML cells. MVs of BMMSC from normal subjects and AML patients were extracted, and the morphology of MVs was observed under electron microscopy to verify the hypothesis. The MVs showed double-concave disk-like particles with a diameter of about $100 \mathrm{~nm}-1 \mu \mathrm{m}$ (Figure 3B). The contents of MV marker proteins CD63, TSG101, HSP70, CD9, and CD81 were detected by western blot (Figure 3C) to verify the successful extraction of MVs. Further detection revealed that the expression of miR-221-3p in the BMMSCderived MVs of AML patients was significantly higher than that of normal subjects (Figure 3D). To identify the delivery of MVs, we labeled BMMSC-derived MVs and OCI-AML2 cells with Dil (red) or Dio (green), respectively. After co-culture, it was observed that Dil spots presented in the OCI-AML2 cells under laser scanning confocal microscope, indicating that the MVs released by the BMMSC were delivered to the OCI-AML2 cells (Figure 3E). Finally, the results of Transwell (Figure 3F) and MTT (Figure 3G) assays showed that the BMMSC-derived MVs significantly improved the migration and invasion abilities as well as cellular activity of OCI-AML2 cells. The results of FCM on co-cultured cells showed that the number of cells was reduced in G0/G1 phase and increased in division stage of the OCIAML2 cell cycle induced by BMMSC-derived MCs (Figure 3H). The expressions of apoptosis-related proteins including PARP, caspase 8 , cleave caspase 8 , and caspase 9 determined by western blot were decreased after the co-culture of BMMSC-derived MVs with OCI-AML2 cells (Figure 3I), indicating that the MVs could 
A

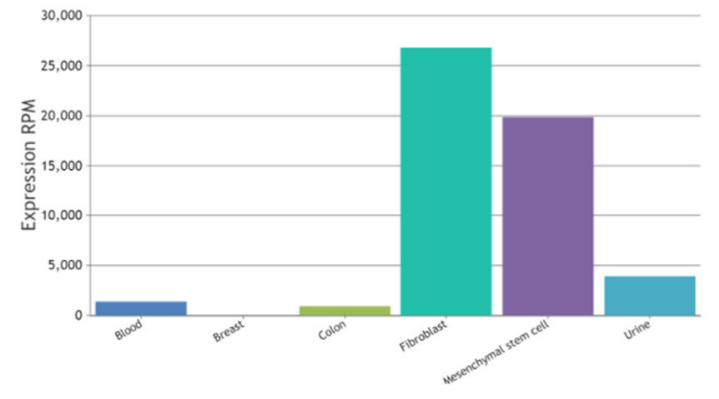

B
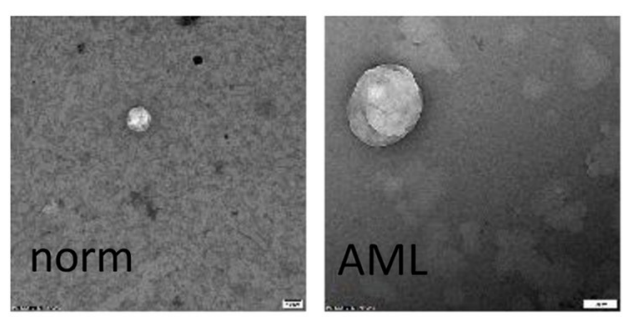

E

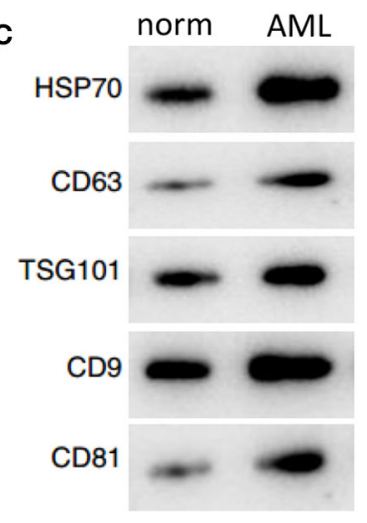

$\mathbf{F}$
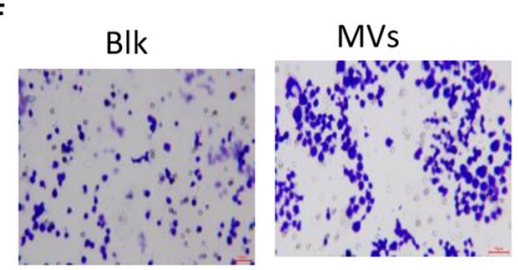

Blk

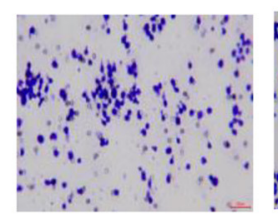

H

Blk

\section{MVs}
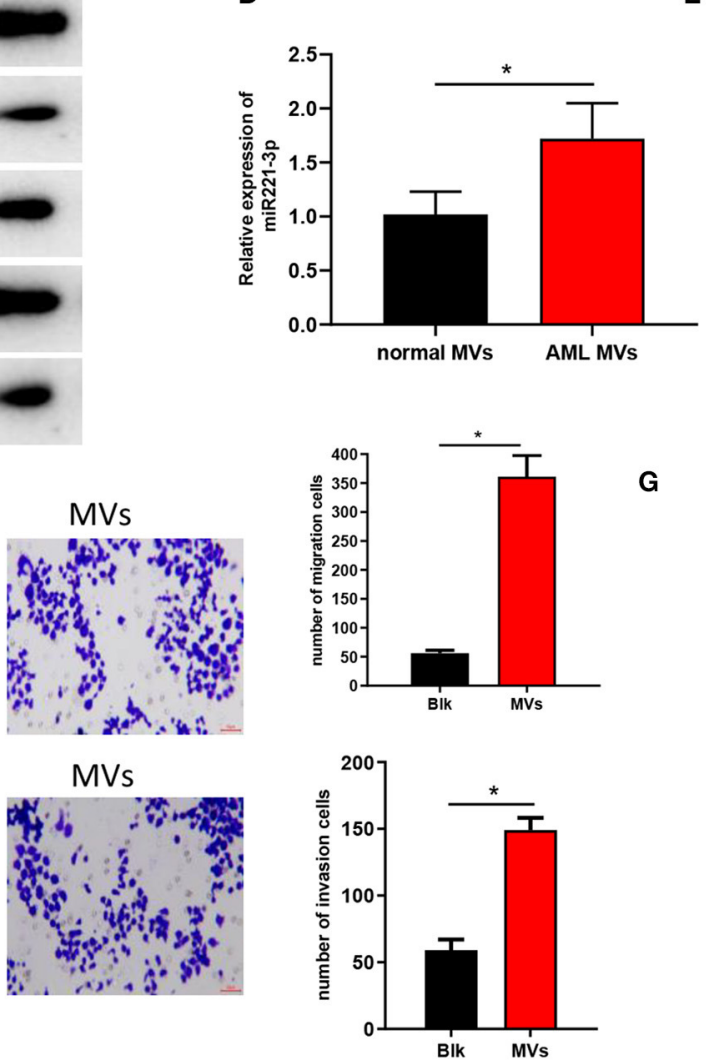

G
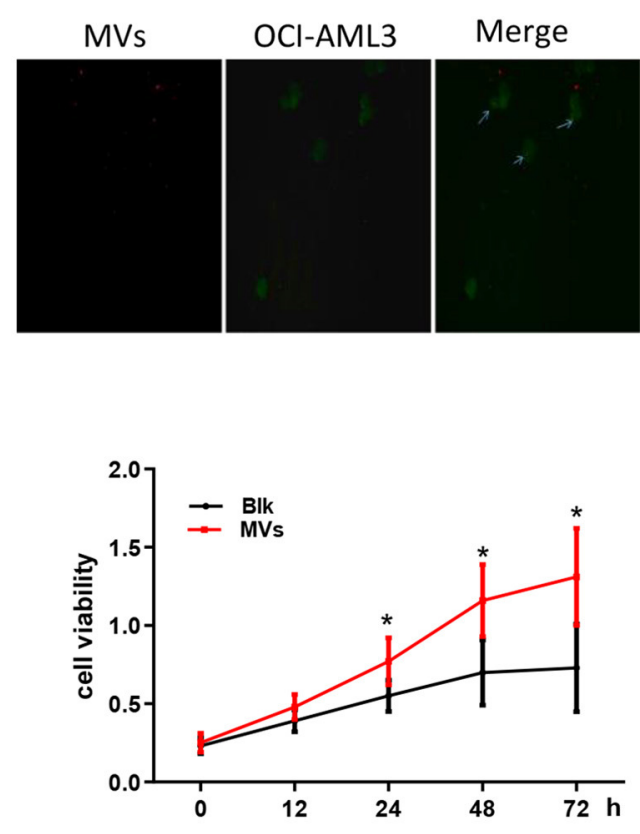

I

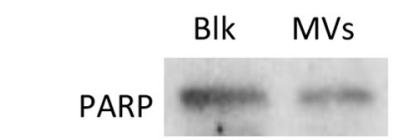

caspase 8

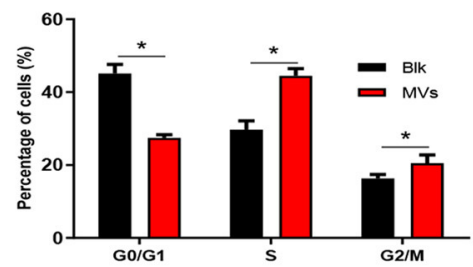

cleave

caspase 8

caspase 9

Actin

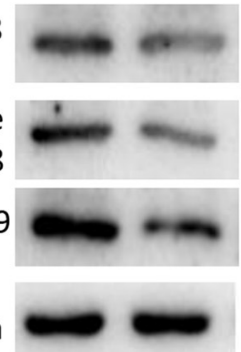

FIGURE 3 | BMMSC-derived MVs regulate AML cell function. (A) The expression of miR-221-3p in the MVs of each cell, the abscissa represents the cell name and the ordinate represents the miRNA expression value. (B) The morphology and size of BMMSC-derived MVs from normal subjects and AML patients were observed under electron microscopy. (C) The changes of MV marker proteins from different sources were detected by western blot. (D) miR-221-3p expression in

BMMSC-derived MVs in normal subjects and AML patients. (E) Fluorescence microscopy showed that Dio-labeled OCl-AML2 cells (green) were transferred to Dil-labeled MVs (red). (F) Transwell assay was conducted to detect the effects of MVs on the invasion and migration ability of OCl-AML2 cells (100x). (G) The effect of MVs on the activity of OCl-AML2 cells was determined by MTT assay. (H) The effects of MVs from different sources on the cell cycle of OCl-AML2 was detected by FCM. (I) The expression changes of apoptosis-related proteins in OCl-AML2 cells affected by MVs from different sources were determined by western blot. 


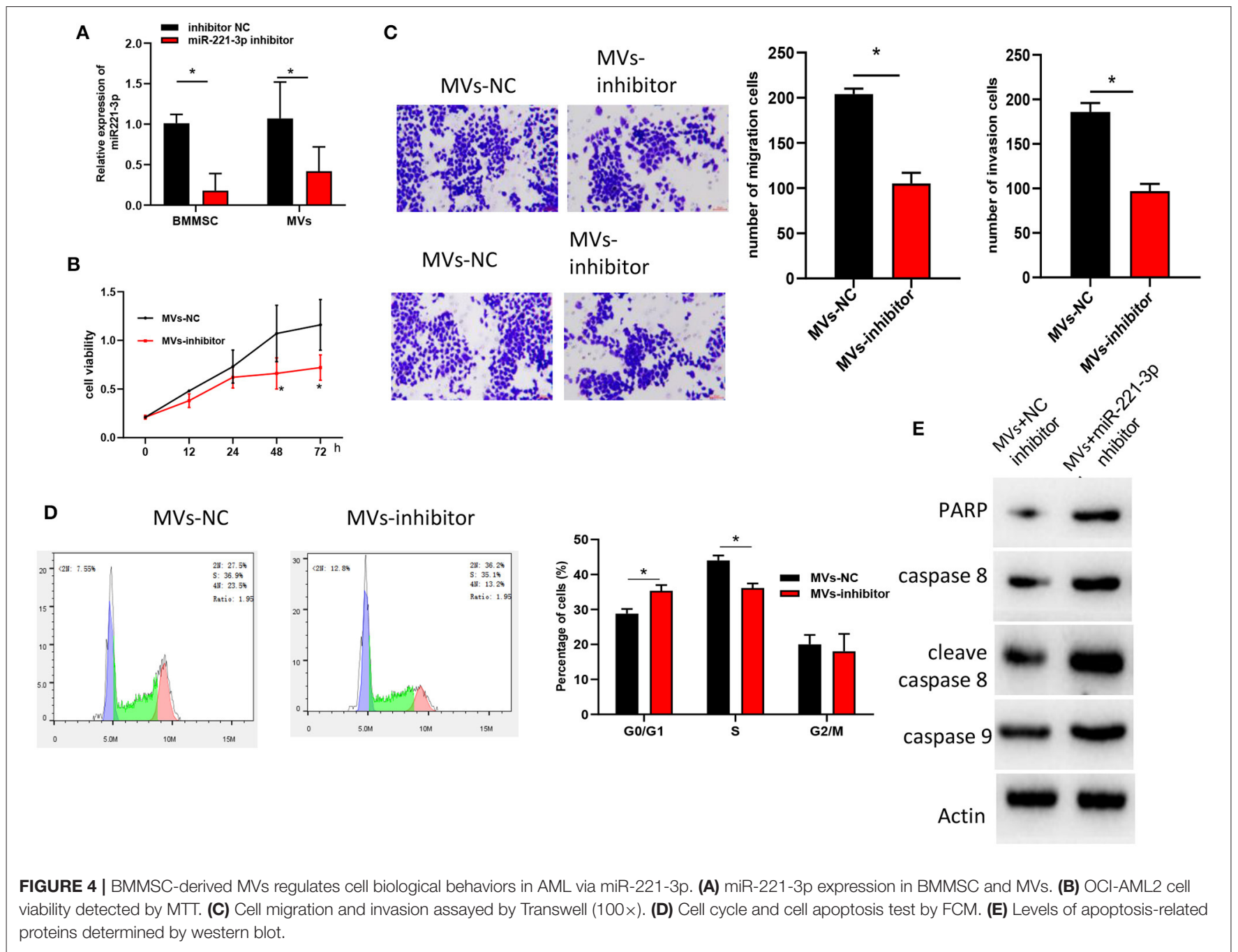

reduce the apoptosis of OCI-AML2 cells, which was in keeping with the results of FCM. These results demonstrated that the BMMSC-derived MVs could enter OCI-AML2 cells, promote the proliferation, migration and invasion, weaken the apoptosis and regulate the cell cycle of OCI-AML2 cells.

\section{BMMSC-Derived MVs Regulates Cell Biological Behaviors in AML via miR-221-3p}

To further investigate the regulatory mechanism of BMMSCderived MVs on OCI-AML2 cell proliferation, invasion and cell cycle via miR-221-3p, inhibitor NC and miR-221-3p inhibitor were transfected into BMMSC, respectively. MVs in two groups were extracted and we found that miR-221-3p was significantly decreased in MVs with miR-221-3p inhibitor relative to that in MVs with NC inhibitor (Figure 4A). Then, the MVs were cocultured with OCI-AML2 cells, showing that MVs in miR-221$3 p$ inhibitor group suppressed the promotive effect of BMMSCderived MVs on cell proliferation, migration and invasion of OCI-AML cells (Figures 4B,C). Meanwhile, FCM revealed that
miR-221-3p inhibitor induced BMMSC cell cycle arrested in G0/G1 phase, indicating that miR-221-3p inhibitor could reverse the effect of BMMSC-derived MVs on cell cycle (Figure 4D). Besides, high expressions of apoptosis-related proteins PARP, caspase 8 , cleave caspase 8 , and caspase 9 were detected by western blot (Figure 4E), and the results suggested that miR-221$3 p$ was capable of abrogating the inhibitory effect of BMMSCderived MVs on OCI-AML2 cells, which was consistent with the FCM results. In all, these findings shed light on that BMMCSderived MVs regulated OCI-AML2 cell biological behaviors via $\mathrm{miR}-221-3 \mathrm{p}$.

\section{miR-221-3p Regulates Cell Proliferation, Invasion and Cell Cycle in AML via Targeting CDKN1C}

The downstream targets of miR-221-3p were predicted by TargetScan, miRSearch and mirDIP databases. Differential analysis was performed on the mRNAs procured from TCGAAML dataset, and eventually 12 potential targets were obtained after the intersection between the identified down-regulated 
A

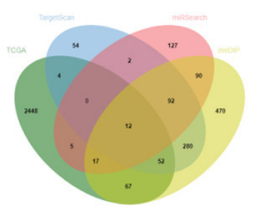

C

3'- U G U U A C A U C G A -5' miR-221-3p

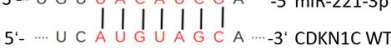

5'- -... U C U A A G U C G A -..-3. CDKN1CMUT

D

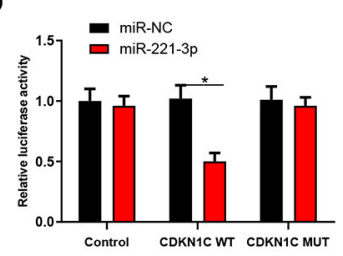

$\mathbf{F}$

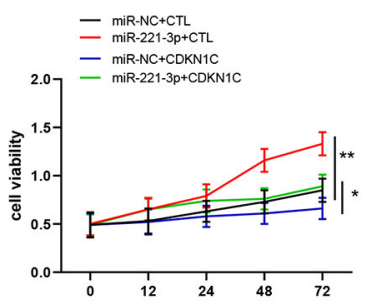

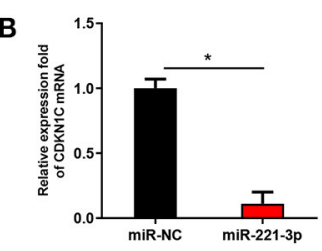

E

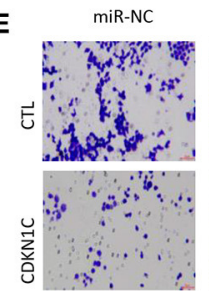

miR-221-3p

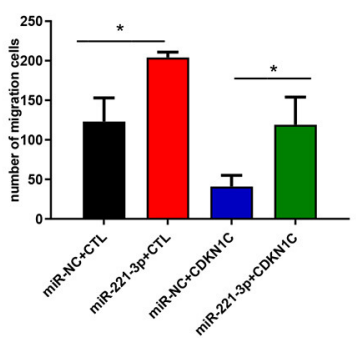

G $\quad$ miR-NC

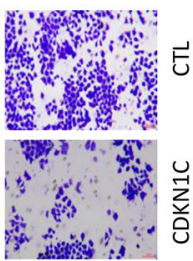

miR-NC

miR-221-3p
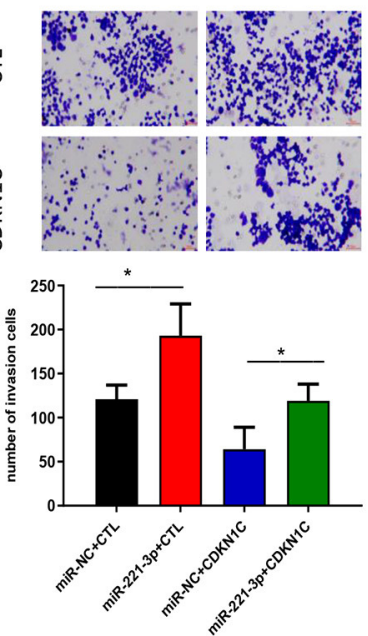

CDKN1C

$\beta$-Actin

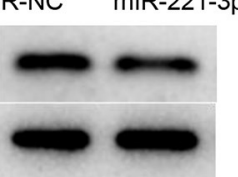

miR-221-3p
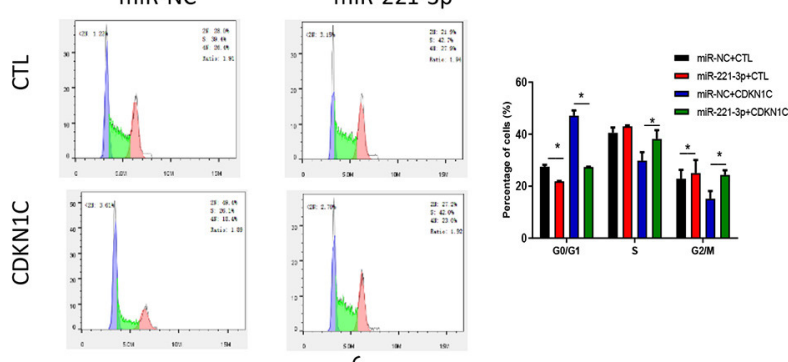

H
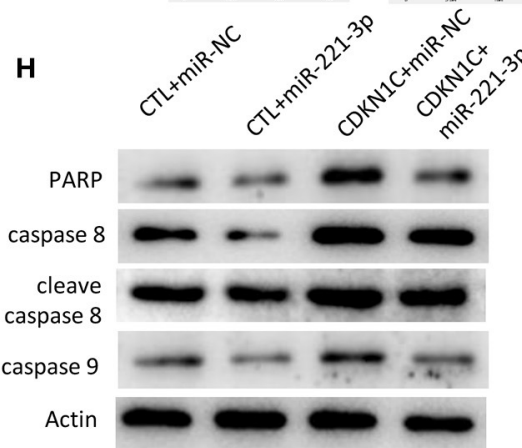

FIGURE 5 | miR-221-3p regulates cell proliferation, invasion and cell cycle in AML via targeting CDKN1C. (A) Venn daigram was plotted to find the potential target genes of miR-221-3p. (B) CDKN1C expression in mRNA and protein levels in the presence of miR-221-3p overexpression. (C) The binding sites of miR-221-3p on CDKN1C 3'-UTR-WT and CDKN1C 3'-UTR-MUT. (D) Relative luciferase activity in each group. (E) Cell migration and invasion detected by Transwell (100x). (F) Cell viability test by MTT. (G) Cell cycle determined by FCM. (H) Protein levels of apoptosis-related proteins measured by western blot.

DEmRNAs and the predicted targets (Figure 5A). Among the 12 target genes, CDKN1C alteration in AML was shown to be the most significant (Table 1). Thereafter, to further validate the relationship between miR-221-3p and CDKN1C, miR221-3p mimic and NC-mimic were, respectively, transfected into OCI-AML2 cells. Western blot and qRT-PCR suggested 
TABLE 1 | Differential expression of the identified 12 potential target genes in the TCGA-AML dataset.

\begin{tabular}{lccccc}
\hline $\begin{array}{l}\text { Gene } \\
\text { symbol }\end{array}$ & Gene ID & $\begin{array}{c}\text { Median } \\
\text { (Tumor) }\end{array}$ & $\begin{array}{c}\text { Median } \\
\text { (Normal) }\end{array}$ & $\begin{array}{c}\text { Log2 (Fold } \\
\text { Change) }\end{array}$ & Adjp \\
\hline CDKN1C & ENSG00000129757.12 & 13.44 & 118.61 & -3.05 & $4.88 E-22$ \\
NRK & ENSG00000123572.16 & 0.93 & 9.153 & -2.395 & $1.77 E-10$ \\
MYLIP & ENSG00000007944.14 & 2.83 & 13.58 & -1.929 & $8.84 \mathrm{E}-26$ \\
POGZ & ENSG00000143442.21 & 11.19 & 31.24 & -1.403 & $1.20 E-27$ \\
FOS & ENSG00000170345.9 & 28.099 & 75.439 & -1.393 & $3.10 E-09$ \\
ARHGEF7 & ENSG00000102606.17 & 9.05 & 23.855 & -1.306 & $4.83 E-36$ \\
CREBZF & ENSG00000137504.13 & 13.06 & 32.565 & -1.255 & $2.01 E-27$ \\
FAM214A & ENSG00000047346.12 & 12.69 & 30.505 & -1.202 & $6.79 E-18$ \\
ADAM22 & ENSG00000008277.14 & 1.37 & 4.23 & -1.142 & $2.88 E-14$ \\
CD4 & ENSG00000010610.9 & 3.35 & 8.185 & -1.078 & $2.74 E-07$ \\
HMBOX1 & ENSG00000147421.17 & 14.57 & 31.545 & -1.064 & $1.92 E-21$ \\
RFX7 & ENSG00000181827.14 & 1.66 & 4.535 & -1.057 & $3.91 E-26$ \\
\hline
\end{tabular}

that the mRNA and protein expressions CDKN1C were reduced in the cells transfected with miR-221-3p mimic (Figure 5B). Then, online miRNA data analysis software (starBase) was applied, finding that miR-221-3p was targeted binding with the CDKN1C $3^{\prime}$-UTR (Figure 5C). Meanwhile, dual-luciferase assay demonstrated that miR-221-3p inhibitory functioned on the luciferase activity in cells transfected with CDKN1C-WT, whereas there was no difference observed in cells transfected with CDKN1C-MUT (Figure 5D). Taken together, we could conclude that CDKN1C was a direct target of miR-221-3p. Subsequently, a series of in vitro experiments were conducted to explore the miR-221-3pdependent mechanism on cell biological behaviors via CDKN1C. Transwell and MTT assays showed that overexpressing of CDKN1C could reverse the promotive role of miR-221-3p overexpression in cell migration, invasion, proliferation and colony forming of OCI-AML cells (Figures 5E,F). Besides, the effects of miR-221-3p overexpression on cell cycle could also be reversed when CDKN1C was simultaneously increased (Figure 5G). Moreover, apoptosis-related proteins were all observed to be elevated after CDKN1C being overexpressed (Figure 5H), elucidating that $\mathrm{CDKN} 1 \mathrm{C}$ overexpression was capable of rescuing the decrease of cell apoptosis induced by miR-221-3p overexpression.

\section{DISCUSSION}

MVs were primarily regarded as unfunctional cellular components to be discarded, yet it has been increasingly suggested that MVs are important tools for the exchange of cellular information and materials, and closely correlated with tumor distant metastasis and immune inhibition (Steinbichler et al., 2017; Fan et al., 2018; Seo et al., 2018; Jerez et al., 2019). MVs are capable of inducing various biological processes after being transferred into recipient cells, such as angiogenesis, metastasis formation, therapeutic resistance, epithelial-mesenchymal transition (EMT) and epigenetic programming (Kreimer et al., 2015; Milane et al., 2015; Gopal et al., 2017). In the present study, we found that miR-221-3p was highly expressed in BMMSC-derived MVs. Besides, it has been reported that bone marrow stromal cell-derived MVs can attenuate the B cell apoptosis in chronic lymphocytic leukemia, also promote cell migration and induce gene expression and modification (Crompot et al., 2017). Hence, this study focused attention on the BMMSC MVs-derived miR-221-3p. Enormous studies have revealed that miR-221-3p is aberrantly expressed in various cancers and participate in the regulation of tumorigenesis and development, like cervical squamous carcinoma (Wu et al., 2019), hepatocellular carcinoma (Li et al., 2019), medulloblastoma (Yang et al., 2019), and breast cancer (Ergun et al., 2015). However, the role of miR-221-3p in AML has not been reported. Therefore, the purpose of this study is to explore the mechanism of miR-221-3p in AML. In our study, we discovered that miR-221-3p was mainly present in BMMSC-derived MVs, and found to be overexpressed in AML patients. Then we constructed miR-221-3p overexpression and found that elevated miR-221-3p was responsible for the promotion of OCI-AML2 cell proliferation, migration and invasion. Moreover, miR-221-3p has been reported to play an important role in other cancers. $\mathrm{Wu}$ et al. have found that miR-221-3p from tumor cell-derived MVs targets THBS2 to facilitate the angiogenesis in cervical squamous carcinoma ( $\mathrm{Wu}$ et al., 2019). Wei et al. have reported that miR-221-3p can potentiate metastasis in cervical cancer via directly targeting THBS2 (Wei et al., 2017). In addition, Shi et al. have revealed that miR-221-3p serving as an oncogene promotively functions on cell proliferation, migration and invasion in gastric cancer through inhibiting PTEN (Shi et al., 2017). Collectively, we believed that miR-221-3p from BMMSC-derived MVs could act as an oncogene beneficial for the cell proliferation, migration and invasion in AML.

In order to further understand the molecular mechanism of miR-221-3p regulating the function of AML cells in BMMSCderived MVs, we proved that miR-221-3p can directly target CDKN1C through bioinformatics analysis and dual-luciferase assay. Besides, there was a negative correlation showed in miR221-3p and CDKN1C expressions both in tissues and cells. CDKN1C is a cyclin-dependent kinase inhibitor $1 \mathrm{C}$, which can inhibit cell proliferation (Adkins and Lumb, 2002; Qiu et al., 2018, 2019). Abnormal expression of CDKN1C plays a role in breast cancer (Qiu et al., 2018), gastric cancer (Sun et al., 2017), glioma (Zhang et al., 2015) and other cancers. And some studies have found that CDKN1C is often methylated in acute lymphoblastic leukemia, and methylation is associated with poor prognosis (Shen et al., 2003). It is found that the expression of $\mathrm{CDKN1C}$ is related to the prognosis of patients with AML (Radujkovic et al., 2016), but the biological function of CDKN1C in AML is unclear. This study found that overexpressing CDKN1C could suppress cell proliferation, migration and invasion in AML. Moreover, CDKN1C was able to reverse the regulation of miR-221-3p overexpression on AML cell biological behaviors when it was concurrently elevated. Taken together, these results suggest that miR-221-3p in BMMSC-derived MVs in AML patients 
regulates the proliferation, invasion, migration and cell cycle by targeting CDKN1C.

In conclusion, our study confirmed that miR-221-3p from BMMSC-derived MVs had the functions of promoting cell proliferation, migration, invasion and regulating cell cycle in AML via targeting CDKN1C. This finding extends our knowledge on the role of miR-221-3p in AML, and helps to further explore the novel approaches for AML targeted therapy.

\section{DATA AVAILABILITY STATEMENT}

The data used to support the findings of this study are included within the article. The data and materials in the current study are available from the corresponding author on reasonable request.

\section{REFERENCES}

Abbasian, N., Herbert, K. E., Pawluczyk, I., Burton, J. O., and Bevington, A. (2018). Vesicles bearing gifts: the functional importance of micro-RNA transfer in extracellular vesicles in chronic kidney disease. Am. J. Physiol. Renal Physiol. 315, F1430-F1443. doi: 10.1152/ajprenal.00318.2018

Adkins, J. N., and Lumb, K. J. (2002). Intrinsic structural disorder and sequence features of the cell cycle inhibitor p57Kip2. Proteins 46, 1-7. doi: $10.1002 /$ prot. 10018

Boyiadzis, M., and Whiteside, T. L. (2018). Exosomes in acute myeloid leukemia inhibit hematopoiesis. Curr. Opin. Hematol. 25, 279-284. doi: 10.1097/MOH. 0000000000000439

Braicu, C., Tomuleasa, C., Monroig, P., Cucuianu, A., Berindan-Neagoe, I., and Calin, G. A. (2015). Exosomes as divine messengers: are they the hermes of modern molecular oncology? Cell Death Differ. 22, 34-45. doi: 10.1038/ cdd.2014.130

Coombs, C. C., Tallman, M. S., and Levine, R. L. (2016). Molecular therapy for acute myeloid leukaemia. Nat. Rev. Clin. Oncol. 13, 305-318. doi: 10.1038/ nrclinonc.2015.210

Cornelissen, J. J., and Blaise, D. (2016). Hematopoietic stem cell transplantation for patients with AML in first complete remission. Blood 127, 62-70. doi: 10.1182/blood-2015-07-604546

Crompot, E., Van Damme, M., Pieters, K., Vermeersch, M., Perez-Morga, D., Mineur, P., et al. (2017). Extracellular vesicles of bone marrow stromal cells rescue chronic lymphocytic leukemia B cells from apoptosis, enhance their migration and induce gene expression modifications. Haematologica 102, 1594-1604. doi: 10.3324/haematol.2016.163337

Del Principe, M. I., Del Principe, D., and Venditti, A. (2017). Thrombosis in adult patients with acute leukemia. Curr. Opin. Oncol. 29, 448-454. doi: 10.1097/ CCO.0000000000000402

Ergun, S., Tayeb, T. S., Arslan, A., Temiz, E., Arman, K., Safdar, M., et al. (2015). The investigation of miR-221-3p and PAK1 gene expressions in breast cancer cell lines. Gene 555, 377-381. doi: 10.1016/j.gene.2014.11.036

Fan, Q., Yang, L., Zhang, X., Peng, X., Wei, S., Su, D., et al. (2018). The emerging role of exosome-derived non-coding RNAs in cancer biology. Cancer Lett. 414, 107-115. doi: 10.1016/j.canlet.2017.10.040

Gopal, S. K., Greening, D. W., Rai, A., Chen, M., Xu, R., Shafiq, A., et al. (2017). Extracellular vesicles: their role in cancer biology and epithelial-mesenchymal transition. Biochem. J. 474, 21-45. doi: 10.1042/BCJ20160006

Hansen, H. P., Engels, H. M., Dams, M., Paes Leme, A. F., Pauletti, B. A., Simhadri, V. L., et al. (2014). Protrusion-guided extracellular vesicles mediate CD30 transsignalling in the microenvironment of Hodgkin's lymphoma. J. Pathol. 232, 405-414. doi: 10.1002/path.4306

Hornick, N. I., Doron, B., Abdelhamed, S., Huan, J., Harrington, C. A., Shen, R., et al. (2016). AML suppresses hematopoiesis by releasing exosomes that contain microRNAs targeting c-MYB. Sci. Signal 9:ra88. doi: 10.1126/scisignal.aaf2797

\section{AUTHOR CONTRIBUTIONS}

XuZ and WY contributed to the study design. YX, JW, SZ, and JL conducted the literature search. $\mathrm{XH}$ and $\mathrm{HX}$ acquired the data. $\mathrm{XiZ}$ and SS wrote the article. YL performed data analysis and drafted. YZ and YX revised the article.

\section{FUNDING}

This study was supported by the funds from National Natural Science Foundation of China (No. 81670124).

\section{SUPPLEMENTARY MATERIAL}

The Supplementary Material for this article can be found online at: https://www.frontiersin.org/articles/10.3389/fbioe. 2020.00081/full\#supplementary-material

Jerez, S., Araya, H., Hevia, D., Irarrázaval, C. E., Thaler, R., van Wijnen, A. J., et al. (2019). Extracellular vesicles from osteosarcoma cell lines contain miRNAs associated with cell adhesion and apoptosis. Gene 710, 246-257. doi: 10.1016/j.gene.2019.06.005

Khwaja, A., Bjorkholm, M., Gale, R. E., Levine, R. L., Jordan, C. T., Ehninger, G., et al. (2016). Acute myeloid leukaemia. Nat. Rev. Dis. Primers 2:16010. doi: $10.1038 /$ nrdp. 2016.10

Kreimer, S., Belov, A. M., Ghiran, I., Murthy, S. K., Frank, D. A., and Ivanov, A. R. (2015). Mass-spectrometry-based molecular characterization of extracellular vesicles: lipidomics and proteomics. J. Proteome Res. 14, 2367-2384. doi: 10.1021/pr501279t

Li, H., Zhang, B., Ding, M., Lu, S., Zhou, H., Sun, D., et al. (2019) C1QTNF1-AS1 regulates the occurrence and development of hepatocellular carcinoma by regulating miR-221-3p/SOCS3. Hepatol. Int. 13, 277-292. doi: 10.1007/s12072-019-09944-5

Lu, X. (2017). The role of exosomes and exosome-derived microRNAs in atherosclerosis. Curr. Pharm. Des. 23, 6182-6193. doi: 10.2174/ 1381612823666170413125507

Milane, L., Singh, A., Mattheolabakis, G., Suresh, M., and Amiji, M. M. (2015). Exosome mediated communication within the tumor microenvironment. $J$. Control Release 219, 278-294. doi: 10.1016/j.jconrel.2015.06.029

Momen-Heravi, F., Bala, S., Kodys, K., Szabo, G., Momen-Heravi, F., Bala, S., et al. (2015). Exosomes derived from alcohol-treated hepatocytes horizontally transfer liver specific miRNA-122 and sensitize monocytes to LPS. Sci. Rep. 5:9991. doi: 10.1038/srep09991

Qiu, Z., Li, Y., Zeng, B., Guan, X., and Li, H. (2018). Downregulated CDKN1C/p57(kip2) drives tumorigenesis and associates with poor overall survival in breast cancer. Biochem. Biophys. Res. Commun. 497, 187-193. doi: 10.1016/j.bbrc.2018.02.052

Qiu, Z., Zhu, W., Meng, H., Tong, L., Li, X., Luo, P., et al. (2019). CDYL promotes the chemoresistance of small cell lung cancer by regulating H3K27 trimethylation at the CDKN1C promoter. Theranostics 9, 4717-4729. doi: 10.7150/thno.33680

Radujkovic, A., Dietrich, S., Andrulis, M., Benner, A., Longerich, T., Pellagatti, A., et al. (2016). Expression of CDKN1C in the bone marrow of patients with myelodysplastic syndrome and secondary acute myeloid leukemia is associated with poor survival after conventional chemotherapy. Int. J. Cancer 139, 1402-1413. doi: 10.1002/ijc.30181

Roversi, F. M., Cury, N. M., Lopes, M. R., Ferro, K. P., Machado-Neto, J. A., Alvarez, M. C., et al. (2019). Up-regulation of SPINT2/HAI-2 by azacytidine in bone marrow mesenchymal stromal cells affects leukemic stem cell survival and adhesion. J. Cell. Mol. Med. 23, 1562-1571. doi: 10.1111/jcmm.14066

Seo, N., Akiyoshi, K., and Shiku, H. (2018). Exosome-mediated regulation of tumor immunology. Cancer Sci. 109, 2998-3004. doi: 10.1111/cas.13735

Shen, L., Toyota, M., Kondo, Y., Obata, T., Daniel, S., Pierce, S., et al. (2003). Aberrant DNA methylation of p57KIP2 identifies a cell-cycle regulatory 
pathway with prognostic impact in adult acute lymphocytic leukemia. Blood 101, 4131-4136. doi: 10.1182/blood-2002-08-2466

Shi, J., Zhang, Y., Jin, N., Li, Y., Wu, S., and Xu, L. (2017). MicroRNA-221-3p plays an oncogenic role in gastric carcinoma by inhibiting PTEN expression. Oncol. Res. 25, 523-536. doi: 10.3727/096504016X14756282819385

Stec, M., Baj-Krzyworzeka, M., Baran, J., Weglarczyk, K., Zembala, M., Barbasz, J., et al. (2015b). Isolation and characterization of circulating micro(nano)vesicles in the plasma of colorectal cancer patients and their interactions with tumor cells. Oncol. Rep. 34, 2768-2775. doi: 10.3892/or.2015.4228

Stec, M., Szatanek, R., Baj-Krzyworzeka, M., Baran, J., Zembala, M., Barbasz, J., et al. (2015a). Interactions of tumour-derived micro(nano)vesicles with human gastric cancer cells. J. Transl. Med. 13:376. doi: 10.1186/s12967-0150737-0

Stein, E. M., and Tallman, M. S. (2016). Emerging therapeutic drugs for AML. Blood 127, 71-78. doi: 10.1182/blood-2015-07-604538

Steinbichler, T. B., Dudás, J., Riechelmann, H., and Skvortsova, I. I. (2017). The role of exosomes in cancer metastasis. Semin. Cancer Biol. 44, 170-181. doi: 10.1016/j.semcancer.2017.02.006

Summerer, I., Niyazi, M., Unger, K., Pitea, A., Zangen, V., Hess, J., et al. (2013). Changes in circulating microRNAs after radiochemotherapy in head and neck cancer patients. Radiat. Oncol. 8:296. doi: 10.1186/1748-717 X-8-296

Sun, C., Ma, P., Wang, Y., Liu, W., Chen, Q., Pan, Y., et al. (2017). KLF15 inhibits cell proliferation in gastric cancer cells via up-regulating CDKN1A/p21 and CDKN1C/p57 expression. Dig. Dis. Sci. 62, 1518-1526. doi: 10.1007/s10620 $-017-4558-2$

Wei, W. F., Zhou, C. F., Wu, X. G., He, L. N., Wu, L. F., Chen, X. J., et al. (2017). MicroRNA-221-3p, a TWIST2 target, promotes cervical cancer metastasis by directly targeting THBS2. Cell Death Dis. 8:3220. doi: 10.1038/s41419017-0077-5
Wu, X. G., Zhou, C. F., Zhang, Y. M., Yan, R. M., Wei, W. F., Chen, X. J., et al. (2019). Cancer-derived exosomal miR-221-3p promotes angiogenesis by targeting THBS2 in cervical squamous cell carcinoma. Angiogenesis 22, 397-410. doi: 10.1007/s10456-019-09665-1

Yang, Y., Cui, H., and Wang, X. (2019). Downregulation of EIF5A2 by miR-221-3p inhibits cell proliferation, promotes cell cycle arrest and apoptosis in medulloblastoma cells. Biosci. Biotechnol. Biochem. 83, 400-408. doi: 10.1080/09168451.2018.1553604

Zhang, J., Gong, X., Tian, K., Chen, D., Sun, J., Wang, G., et al. (2015). miR-25 promotes glioma cell proliferation by targeting CDKN1C. Biomed. Pharmacother. 71, 7-14. doi: 10.1016/j.biopha.2015.02.005

Zheng, H., Liu, J. Y., Song, F. J., and Chen, K. X. (2013). Advances in circulating microRNAs as diagnostic and prognostic markers for ovarian cancer. Cancer Biol. Med. 10, 123-130. doi: 10.7497/j.issn.2095-3941.2013.03.001

Zhou, C. F., Ma, J., Huang, L., Yi, H. Y., Zhang, Y. M., Wu, X. G., et al. (2019). Cervical squamous cell carcinoma-secreted exosomal miR-221-3p promotes lymphangiogenesis and lymphatic metastasis by targeting VASH1. Oncogene 38, 1256-1268. doi: 10.1038/s41388-018-0511-x

Conflict of Interest: The authors declare that the research was conducted in the absence of any commercial or financial relationships that could be construed as a potential conflict of interest.

Copyright (c) 2020 Zhang, Xu, Wang, Zhao, Li, Huang, Xu, Zhang, Suo, Lv, Zhang and $Y u$. This is an open-access article distributed under the terms of the Creative Commons Attribution License (CC BY). The use, distribution or reproduction in other forums is permitted, provided the original author(s) and the copyright owner(s) are credited and that the original publication in this journal is cited, in accordance with accepted academic practice. No use, distribution or reproduction is permitted which does not comply with these terms. 